\title{
Should I Go With My Gut? Investigating the Benefits of Emotion-Focused Decision Making
}

\author{
Joseph A. Mikels \\ DePaul University
}

\author{
Andrew E. Reed \\ Stanford University
}

\author{
Sam J. Maglio \\ New York University
}

Lee J. Kaplowitz

State University of New York at Buffalo

\begin{abstract}
Deliberative decision strategies have historically been considered the surest path to sound decisions; however, recent evidence and theory suggest that affective strategies may be equally as effective. In four experiments we examined conditions under which affective versus deliberative decision strategies might result in higher decision quality. While consciously focusing on feelings versus details, participants made choices that varied in complexity, in extent of subsequent conscious deliberation allowed, and in domain. Results indicate that focusing on feelings versus details led to superior objective and subjective decision quality for complex decisions. However, when using a feeling-focused approach, subsequent deliberation after encoding resulted in reduced choice quality. These results suggest that affective decision strategies may be more effective relative to deliberative strategies for certain complex decisions.
\end{abstract}

Keywords: gut feeling, intuition, emotion, decision making

Modern life is inundated with complex decisions, from purchasing a car and deciding where to go on vacation to making decisions about physicians and medical treatments. When making such decisions, people sometimes hold the various factors in memory and deliberate over them in order to make the best possible choice. However, at other times people focus on their feelings regarding the options and ultimately "go with their gut." The deliberative approach has traditionally been considered the best path in decision making (see, e.g., Janis \& Mann, 1977), but introspection about a choice has been shown to lead to less optimal decisions (see, e.g., Wilson et al., 1993; Wilson \& Schooler, 1991). Moreover, recent evidence suggests that using affect as a heuristic in decision making may be an equally important path (for a review see Slovic, Finucane, Peters, \& MacGregor, 2002)—and may even be critical to some decisions (Damasio, 1994). Here we report four studies examining the conditions under which affective decision strategies could potentially be beneficial, with data supporting the notion that focusing on feelings versus details during information acquisition can improve the quality of certain decisions.

Descriptive decision research and theory has focused heavily on the cognitive, deliberative aspects of decision making. However,

This article was published Online First May 30, 2011.

Joseph A. Mikels, Department of Psychology, DePaul University; Sam J. Maglio, Department of Psychology, New York University; Andrew E. Reed, Department of Psychology, Stanford University; Lee J. Kaplowitz, School of Medicine, State University of New York at Buffalo.

This research was supported, in part, by the Decision, Risk and Management Sciences program at the National Science Foundation, Research Grant SES-1023206.

Correspondence concerning this article should be addressed to Joseph A. Mikels, Department of Psychology, DePaul University, 2219 N. Kenmore Avenue Chicago, IL 60614. E-mail: jmikels@ depaul.edu the efficacy of cognitive processes should not be evaluated in isolation-one should also consider the influence of affective processes, as suggested by dual process theories of decision making. Dual process theories draw the distinction between two general processing streams that lead individuals to make their choices: intuitive and deliberative (e.g., Epstein, 1994; Kahneman, 2003; Loewenstein, Weber, Hsee, \& Welch, 2001; Reyna, 2004; Stanovich \& West, 2000). The intuitive system (system 1) is considered to be experiential and is generally characterized as quick, automatic, gist-based, and affective. In contrast, the deliberative system (system 2) is considered to be generally slow, controlled, verbatim-based, and analytic in nature. Although the distinctions between systems 1 and 2 themselves are a useful heuristic, it is becoming increasingly clear that such an overarching dichotomy is oversimplified and cannot coherently accommodate all proposed distinctions (for a review see Evans, 2008). For instance, whereas some dual process models include affect centrally within the intuitive system (see, e.g., Epstein, 1994), others do not include affect and consider the intuitive system to be entirely cognitive and implicit in nature (see Evans, 2008 for a review). Hence, greater precision is necessary.

The present line of research takes into account a dual process model that includes affect as a central component: cognitiveexperiential self-theory (CEST; Epstein, 1994). When individuals are faced with decisions, there are multiple sources of information to consciously consider including "hot" and "cold" cognitions. The former stream of information is emotional in nature, whereas the latter involves "rational" and deliberative processes (see, e.g., Janis \& Mann, 1977). According to CEST, behavior and decisions are guided by both affect-laden experiential and rational-analytic parallel systems (see Epstein, 1994; Epstein, Pacini, Denes-Raj, \& Heier, 1996). Critical to the current studies, the experiential system 
is intuitive in nature and intimately associated with affect. Importantly, previous research has shown that differential reliance on affect versus deliberation can lead to different judgments (Hsee \& Rottenstreich, 2004). Moreover, research suggests that there are no overarching principles that govern the benefits of affective versus deliberative processes (e.g., Shiv, Loewenstein, Bechara, Damasio, \& Damasio, 2005). As such, the current approach attempts to disentangle some of the boundary conditions, while also offering a different dual-process approach in distinguishing the contributions of conscious deliberative versus affective processes in decision making.

Although both modes of processing play an important role in the decision making process, reliance on the "intuitive" system-via various heuristics - has historically been shown to lead to flawed decisions (for reviews see Gilovich, Griffin, \& Kahneman, 2002). However, more recently the potential benefits of the intuitive system have been highlighted (Kahneman, 2003; Reyna, 2004; Slovic et al., 2002), in addition to evidence for the negative effects of deliberation on decision quality (e.g., Wilson \& Schooler, 1991). In related research, individuals who have a high level of skill or expertise within a given domain appear to rely to a greater extent on intuitive judgments, implying that intuition is an advanced means of decision making (see, e.g., Reyna, 2004; Reyna \& Lloyd, 2006). Thus, although research in decision making has revealed many pitfalls of intuitive judgments (e.g., Gilovich et al., 2002), it remains unclear the extent to which intuitive relative to deliberative processes may be beneficial to decisions. More precise process-level distinctions may provide greater clarity-specifically in the operational distinction of "intuitive" processing as the conscious processing of the emotional aspects of decision attributes.

\section{The Affect Heuristic}

Certain lines of theorizing about the intuitive system have emphasized the role of affective processing in decision making (for a review see Loewenstein et al., 2001; Slovic et al., 2002). For instance, Slovic and colleagues have introduced a theoretical framework that explicates how decision alternatives are "tagged" with differing degrees of positive and negative affect: the affect heuristic (see Finucane, Alhakami, Slovic, \& Johnson, 2000; Peters, Hess, Vastfjall, \& Auman, 2007; Slovic et al., 2002; Slovic, Peters, Finucane, \& MacGregor, 2005). The affect heuristic can be used to explain various findings in judgment and decision making such as the evaluability of options (e.g., Hsee, 1996), proportion dominance (e.g., Hsee, 1998), risk and benefit judgments (Alhakami \& Slovic, 1994; Finucane et al., 2000), the ratio bias (Denes-Raj \& Epstein, 1994), and so forth. In addition, a large body of evidence suggests that emotional reactions play a major role in risky decision making - that is, choice under conditions of risk or uncertainty - as explicated by the affect heuristic as well as in the risk-as-feelings hypothesis (Loewenstein et al., 2001). The lion's share of evidence indicates that these affective influences may lead to poor decisions (see above); however, as implicated by the affect heuristic, "using an overall, readily available affective impression can be far easier-more efficient - than weighing the pros and cons or retrieving from memory many relevant examples, especially when the required judgment or decision is complex or mental resources are limited" (Slovic et al., 2002, p. 400). Thus, affective decision processes may be beneficial for complex decisions when time to consciously process the attributes is limited. In contrast, with adequate time to consciously process the details, a deliberative approach may be beneficial. Nonetheless, although affect is theorized to be an efficient route to good decisions, the precise benefits remain speculative.

A dramatic example of the crucial role of affect in risky decision making comes from the work of Damasio and colleagues (see, e.g., Damasio, 1994), who have documented that patients with damage to the ventromedial prefrontal cortex make severely flawed and nonoptimal decisions. In an extensive series of observations and studies, it has been demonstrated that the nonoptimal decisions of these patients resulted from their inability to use anticipatory feelings to guide behavior and decisions despite preserved intellectual and deliberative abilities. While these various lines of research and theory emphasize affective along with deliberative processes within the decision making process, the circumstances under which affective processing may be beneficial to decision making require further delineation. Thus, empirical investigation of such circumstances would not only extend previous work on the affect heuristic, but also extend the ecological rationality approach, which emphasizes the importance of understanding the heuristicenvironment fit (Gigerenzer, 2008; Todd \& Gigerenzer, 2007).

Importantly, the introduction of the affect heuristic suggests a concrete and specific distinction between two dual processes that involve a focus on feelings versus details during the decision making process. Whereas a general dual process model draws important broad distinctions, the specific contrast of using feelings versus details for decisions allows for a precise distinction that can be operationally defined. That is, reliance on processes that encode affective impressions versus the details into working memory would allow for a precise test of the efficacy of these dual processes. Such an approach has previously been useful in revealing age differences in decision making; although older adults fared as well as younger adults using a feeling-focused strategy, reliance on a detail-focus strategy impaired the performance of older adults relative to their younger counterparts (Mikels et al., 2010). Moreover, in support of the notion that emotion-based information can be stored in parallel with detail-based information, recent work suggests that there may be separable working memory subsystems for emotional versus nonemotional information (Mikels, ReuterLorenz, Beyer, \& Fredrickson, 2008). As such, the central goal of the current project was to examine the differential decision outcomes of feeling versus detail focused encoding into working memory as informed by the attitude formation literature.

\section{Affective Versus Cognitive Focus in Attitude Formation}

Although we know of very limited research directly examining the impact of focus on decision outcomes, the distinction between affective and cognitive processes in attitude formation has a long and prominent history. This line of research and theory importantly underscores that there are distinct and separable affective and cognitive components of attitudes (Breckler, 1984; Breckler \& Wiggins, 1989; Trafimow \& Sheeran, 1998), and that attitudes can be experimentally created that are either based more or less on affective versus cognitive factors (Crites, Fabrigar, \& Petty, 1994). For example, one prominent set of manipulations involve directing 
the focus of attention to either affective versus cognitive aspects of the attitude object, resulting in attitudes that are drawn more strongly from affective versus cognitive factors, respectively (e.g., Millar \& Tesser, 1986; van den Berg, Manstead, van der Pligt, \& Wigboldus, 2006).

For instance, van den Berg et al. (2006) found that participants who were induced to focus on affect constructed attitudes that were congruent with affective versus cognitive information about an object, whereas those induced to focus on cognition and reasoning formed the opposite attitudes. In other words, manipulating the focus of attention to affective or cognitive factors of an attitude object can result in the formation of different attitudes despite being exposed to the same information. Additionally, focusing on feelings versus thoughts regarding an attitude object has been shown to differentially correspond to hedonic versus instrumental behavior (Millar \& Tesser, 1986). Such findings suggest that emphasizing the affective versus cognitive component of an attitude not only influences the attitude itself but also subsequent behavior. Extending this line of research into the decision domain, it then follows that focusing on affective versus cognitive aspects during attitude formation could subsequently influence the ultimate quality of a decision. However, to our knowledge, such conjectures have heretofore gone uninvestigated.

\section{Unconscious Processes and Decision Making}

Despite the lack of direct research into the benefits of affective focus on decision outcomes, related work on unconscious processing in decision making provides a useful point of comparison. In a manner consistent with-but different fromdual process theories, Dijksterhuis and Nordgren's (2006) unconscious-thought theory (UTT) distinguishes between two modes of thought: conscious and unconscious. The theory holds, for instance, that conscious thought has limited capacity and is top-down, whereas unconscious has high capacity and is bottom-up. With respect to the dual process models described above, UTT differs in several important ways (see Dijksterhuis \& Nordgren, 2006). For instance, UTT predicts that relatively effortless unconscious thought can lead to more desirable outcomes in contrast to effortful conscious thought. This postulate runs contrary to other dual process models, which predict superior outcomes via effortful processes. Additionally, UTT does not perfectly match the deliberative and intuitive systems that many dual process models invoke, as UTT contends that both conscious and unconscious processes are able to work on the same inputs. Thus, although UTT resembles other dual process models, there are important theoretical distinctions.

Although UTT has been considered relevant to various psychological processes, the application of the theory to decision making has been notably disputed. The theory holds that conscious thought is superior for simple decisions but that unconscious thought is superior for complex decisions, as demonstrated in multiple studies (e.g., Dijksterhuis, 2004; Dijksterhuis, Bos, Nordgren, \& van Baaren, 2006). In these studies, participants are presented with either simple decisions (typically involving four pieces of information for four options) or complex decisions (typically involving 12 pieces of information for four options). For the simple decisions, participants who are given subsequent time to deliberate after information acquisition make higher quality decisions rela- tive to participants who are distracted for an equal amount of time following information acquisition. In stark contrast, for complex decisions, participants make inferior choices when given subsequent time to think about the decision, relative to participants who are distracted. Importantly, this latter condition also results in superior decisions in comparison to conditions in which participants make a decision immediately, suggesting that participants engage in productive "unconscious" deliberation about the decision during distraction.

Nevertheless, despite the allure of the above findings, UTT remains controversial. For instance, more recent work has not replicated these results - possibly due to various moderating factors such as presentation format (see, e.g., Acker, 2008). Moreover, findings in cognitive and social psychology have called the tenets of UTT into question (see, e.g., González-Vallejo, Lassiter, Bellezza, \& Lindberg, 2008). For instance, recent work by Lassiter, Lindberg, Gonzalez-Vallejo, Bellezza, and Phillips (2009) suggests that the benefits of unconscious deliberation are due to online working memory-based judgments versus long-term memory-based judgments. The specific nature of these online processes, though, remains opaque but may be related to configural versus featural processing. Specifically, unconscious processes during distraction led to superior decisions with initially encoded configural holistic impressions but not featural detailbased impressions (Lerouge, 2009). The broad distinction between configural versus featural impressions parallels the distinction between feeling- versus detail-based focus suggested by the affective heuristic.

Most critical to the current investigation, UTT distinguishes between conscious and unconscious thought, but with both involving "object-relevant or task-relevant cognitive or affective thought processes" (p. 96) that occur after information acquisition (Dijksterhuis \& Nordgren, 2006). UTT also draws linkages between unconscious thought, affect, and intuition insofar as intuition is considered "a gut feeling based on unconscious past experience" (p. 105), which raises the possibility that the superiority of unconscious thought for complex decisions may be partially due to reliance on affective processing. Although intuitive decision processes need not be conceptualized as intrinsically affective in nature, some theoretical perspectives place affect centrally within the purview of the experiential/intuitive system (e.g., Epstein, 1994; Slovic, Finucane, Peters, \& MacGregor, 2004).

These speculations raise intriguing connections with the affect heuristic but with a few critical theoretical distinctions. In particular, the affect heuristic distinguishes between the conscious affective and cognitive processes at work during decision making with minimal consideration of unconscious processes (Slovic et al., 2002). Additionally, the affect heuristic considers the role of emotion during the entire decision process-from encoding to final choice-whereas UTT largely focuses on processes at work after encoding. Thus, conscious focus on feelings versus the details at encoding would allow for a direct test how affective versus cognitive processes may benefit decisions outcomes as predicted by the affect heuristic literature. It remains unclear-yet intriguing-how subsequent conscious versus unconscious thought may interface with initial affective versus cognitive information acquisition. Thus, although the conscious versus unconscious distinction 
resembles the deliberative versus affective distinction, they are indeed orthogonal.

\section{Present Investigation}

In order to examine whether affective versus deliberative processing may be more effective for complex decisions, we manipulated the encoding strategies individuals used during various decision tasks. As both affective and deliberative processes contribute to decisions naturally, our intent was to examine whether strategic reliance on affective versus deliberative processing could result in superior decisions as suggested by theorizing about the affect heuristic (e.g., Slovic et al., 2002). We operationalized affective and deliberative processing as the differential encoding of decision options into working memory via attentional focus on feelings versus details during information acquisition. As described above, this operational definition is derived from the affect heuristic and attitude formation literatures, which in turn provides a specific contrast with which the benefits of affect to decision making can be better specified. That is, manipulations of initial attentional focus influence attitudes and subsequent behavior (e.g., Millar \& Tesser, 1986; van den Berg et al., 2006), and in a decision context could impact decision quality.

Our emphasis on encoding as a direct way to test the affect heuristic raises critical methodological and theoretical considerations. In the work of Dijksterhuis and colleagues (e.g., 2004; 2006) based on UTT, the experimenters manipulated the availability of conscious versus unconscious thought following information acquisition. In the present work, we manipulated how participants consciously encoded the information pertaining to the decisions into working memory. The decision process involves multiple stages, from information acquisition and encoding to subsequent deliberation and then the ultimate choice, during which affective and cognitive processes are ostensibly at work. As a critical test of the affect heuristic, we sought to disentangle the effects of conscious affective versus cognitive processes on choice quality at the initial stage of information acquisition. Although our approach complements that of Dijksterhuis and colleagues, it addresses the orthogonal constructs of affective versus cognitive processing.

In the first two studies, we manipulated focus on feelings versus details as information was encoded into working memory in a decision task and then examined subsequent decision quality for simple versus complex decisions (Experiment 1) and with consideration of objective and subjective decision quality (Experiment 2). In Experiment 3, we sought to examine how subsequent conscious versus unconscious thought may influence the quality of decisions that are initially encoded affectively versus cognitively. Finally, in Experiment 4, we sought to extend our approach using a different methodology and additional domains.

\section{Experiment 1}

In the first study, we sought to directly test the efficacy of the affect heuristic for relatively simple versus complex decisions as operationally defined above. As such, we manipulated attentional focus as information was encoded into working memory by devising two conditions - one in which participants focused on their feelings about the options, and a second in which participants focused on the details of the options. We also manipulated the complexity of the decision to examine whether a focus on feelings would lead to higher decision quality. Based on theorizing about the affect heuristic, we predicted that focusing on feelings versus details would benefit complex but not simple decisions.

\section{Method}

\section{Participants}

Seventy-six undergraduates $(M$ age $=19.24$ years; 54 females, 22 males) were randomly assigned to conditions in a 2 (feeling vs. detail focus) $\times 2$ (simple vs. complex decisions) betweenparticipants design. They received course credit for participation.

\section{Apparatus}

Stimuli were presented to participants on a 17" LCD screen using a Dell Optiplex GX270 desktop with E-Prime experimental software. Participant responses were recorded via a standard keyboard.

\section{Design and Procedure}

The procedure and stimuli were adapted from Study 1 of Dijksterhuis et al. (2006). The strategy manipulations were based on those used by Mikels et al. (2010). Participants were presented with information about four hypothetical cars. Information was presented one attribute at a time (serially) and in random order for 4 seconds per attribute. Each attribute was positive (e.g., "gets good gas mileage") or negative (e.g., "gets poor gas mileage"). The options were designed such that one car (the "best" option) had $75 \%$ positive attributes, two cars had $50 \%$ positive attributes, and one car had $25 \%$ positive attributes.

To manipulate strategy, we devised two between-participants conditions: feeling-focus and detail-focus. Before reading the information about the options, participants were instructed to attend to, and base their decision on, either their feelings about the options or the details about the options (depending on condition). Additionally, strategy manipulations were reinforced after each attribute was presented: participants responded to queries regarding either their feelings or their memory for the corresponding car. Participants in the feeling-focus condition reported how they felt about the corresponding option on a 7-point Likert-type scale (from 1-very negative to 7-very positive). Participants in the detail-focus condition reported how well they were remembering the option on a 7-point Likert-type scale (from 1- not at all to 7very well). Importantly, the instructions and query manipulation focused on the actual options-rather than on the specific attributes-so as to target working memory processes rather than long-term memory processes.

To manipulate complexity, we constructed two betweenparticipants conditions: simple and complex decisions. Half of our participants (randomly assigned) viewed information about four hypothetical cars, each defined by four attributes (simple condition). The other half of the participants viewed information about four hypothetical cars as well, but defined by 12 attributes each (complex condition). The attributes for each car were alignablethat is, each car was described by the same attributes but either 
positively or negatively. After viewing all of the information, participants were asked to choose a car.

After several unrelated additional questionnaires, participants completed a free-recall task in which they wrote down as many of the details they could remember for each car. Finally, to control for the subjective importance of attributes, we then had participants rate the importance of each attribute (e.g., "whether a car gets good gas mileage"). Participants rated each attribute on a 7-point Likerttype scale (from 1-not important at all to 7-very important). These importance ratings were collected to weigh the options for each participant individually, with which an alternate scoring system was used to score choice quality.

\section{Results and Discussion}

To examine decision quality in each condition, we compared the number of participants who chose the "best" option (the car with $75 \%$ positive attributes) using chi-square tests. As depicted in Figure 1, for simple decisions the number of participants in the feeling-focus condition that chose the best option (31.6\%) did not differ from those in the detail-focus condition $(47.4 \%), \chi^{2}(1, N=$ $38)=1.81, n s$. However, for complex decisions significantly more participants in the feeling-focus condition $(68.4 \%)$ chose the best option relative to those in the detail-focus condition $(26.3 \%), \chi^{2}(1$, $N=38)=5.16, p<.05$. Importantly, when we recoded the "superiority" of the options with respect to each participant's importance ratings for each attribute, the results remained entirely unchanged, thus confirming our a priori designations. Although such measures provide converging support for the designations, it remains possible that the choice participants made influenced the importance ratings as suggested by evidence that choices can influence evaluations (for a review see, e.g., Brownstein, 2003).

In order to examine whether the conditions resulted in differential long-term memory encoding, we conducted an ANOVA on the percentage of attributes recalled with focus (details, feelings) and complexity (simple, complex) as between-participants factors. The only significant effect was the main effect of complexity, $F(1$,

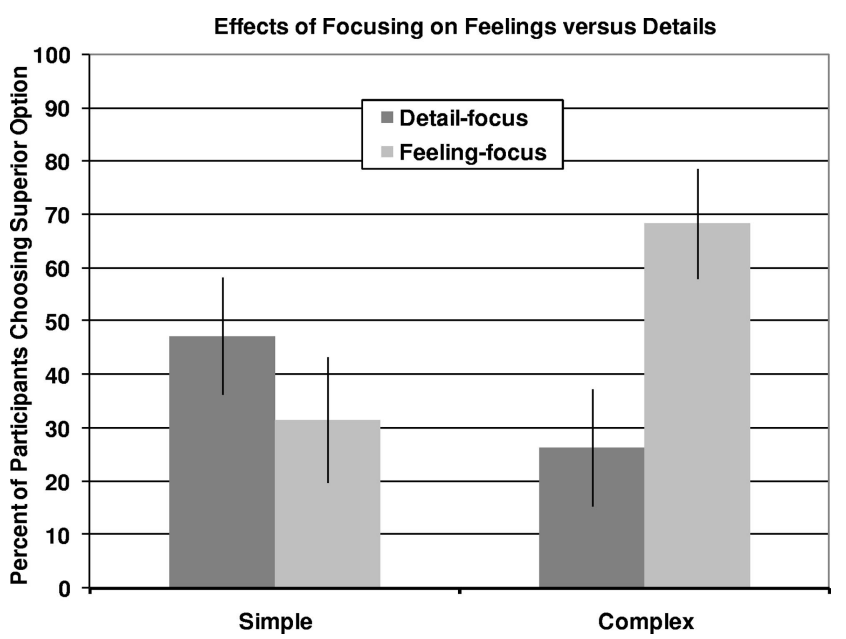

Figure 1. Percentage of participants in Experiment 1 who chose the best option (the car with $75 \%$ positive attributes) in each condition. Error bars represent the standard error.
72) $=33.08, p<.001, \eta_{\mathrm{p}}^{2}=.32$. Thus, although participants in the simple conditions recalled a higher percentage of attributes relative to the complex condition (for means see Table 1), the manipulation of focus did not influence long-term memory recall suggesting that the focus manipulations successfully altered encoding into working memory rather than long-term memory encoding.

In this study we set out to examine whether a focus on feelings versus details would result in higher choice quality for complex decisions, and found precisely that. Importantly, an alternative possibility as suggested by the work of Lassiter et al. (2009) is that the detail-focus condition relied on memorization and encoding into long-term memory. However, given the lack of a difference in recall between the focus conditions, it is unlikely that our manipulation recruited long-term memory differentially. Thus, encoding feelings into working memory resulted in superior objective choice quality relative to encoding the details into working memory. But does such an encoding focus extend to subjective aspects of choice quality? In Experiment 2, we sought to further address these differences by extending the effects to subjective decision quality.

\section{Experiment 2}

Experiment 1 provided evidence that focusing on feelings versus details can result in higher objective choice quality (i.e., accuracy) for complex decisions, but it remains unclear whether the benefits of such a focus extend to subjective choice quality (i.e., satisfaction and confidence). Moreover, the choices were constructed such that there was a dominating alternative, which was predetermined as the "best" choice. To complement this design and better assess the subjective dimensions of choice quality, we employed a design without an a priori dominating alterative.

In Experiment 2, we further explore the benefits of focusing on feelings by examining subjective choice quality for a choice set without a dominating alternative. Additionally, using importance ratings from each participant, we determined the "best" choice for each participant and provide an alternative measure of objective choice quality. Finally, we measured participants' postchoice recall of attributes to examine if the feeling versus detail focus condition resulted in superior long-term memory for the attributes. We contend that the benefits to objective choice quality of focusing on feelings versus details during decision making would extend to subjective choice quality and are unique to affective encoding rather than deeper processing-thus resulting in commensurate levels of recall.

\section{Method}

\section{Participants}

Forty undergraduates $(M$ age $=19.78$ years; 34 females, 6 males) were randomly assigned to either a feeling-focus or a detail-focus condition. They received course credit for participation.

\section{Apparatus}

Same as Experiment 1. 
Table 1

Percentage of Recalled Attributes by Strategy and Decision Complexity for Experiment 1

\begin{tabular}{lccc}
\hline & \multicolumn{2}{c}{ Strategy } & \\
\cline { 2 - 3 } Complexity & Feeling-focus & Detail-focus & Average \\
\hline Simple & $39.5(2.6)$ & $36.9(3.4)$ & $38.2(3.0)$ \\
Complex & $22.1(1.8)$ & $24.5(2.3)$ & $23.3(2.1)$ \\
Average & $30.8(2.2)$ & $30.7(2.9)$ & \\
\hline
\end{tabular}

Note. Standard errors are in parentheses.

\section{Design and Procedure}

The stimuli and procedure were very similar to Experiment 1 : participants were either assigned to a feeling-focus or a detailfocus condition, but in this experiment only made complex decisions (four hypothetical cars, each defined by 12 attributes). In contrast to Experiment 1, the options in this study were designed such that there was no dominating alternative; each of the four cars was defined by $50 \%$ positive attributes and $50 \%$ negative attributes. Moreover, using the importance rating data for the attributes from Experiment 1, we constructed the options such that each car was relatively matched overall in the distribution of normative importance for its positive versus negative attributes.

In this experiment, we included several additional measures that were not included in Experiment 1. First, after participants chose a car, they then rated their satisfaction with their choice on a 7-point Likert-type scale, with 1 indicating that they were not at all satisfied and 7 indicating that they were extremely satisfied. They also rated how confident they were that they made the best possible choice on a 7-point Likert-type scale with 1 indicating that they were highly unconfident and with 7 indicating that they were highly confident. In addition, following these ratings of subjective choice quality, participants completed a surprise recall test for the attributes in which they were instructed to write down everything they remembered about each car on a sheet of paper listing the four car names. Finally, we also collected subjective importance ratings for each attribute in the same manner as described in Experiment 1.

\section{Results and Discussion}

In order to assess subjective choice quality, independent sample $t$ tests were conducted on the satisfaction and confidence measures. Supporting the notion that focusing on feelings versus details would result in increased subjective choice quality, participants in the feeling-focus condition were more satisfied with and confident about their choice than those in the detail-focus condition, $t(38)=$ $2.54, p<.05 \& t(38)=2.28, p<.05$, respectively (see Figure 2 ).

Although the choice options were constructed such that each consisted of the same percentage of positive and negative attribute roughly equated on normative importance, using the importance ratings from each participant in this experiment, we were able to determine the "best" option for many of the participant. Specifically, we calculated a weighted score for each car by summing the importance rating of each positive and negative attribute, which resulted in the detection of a moderately superior option for 36 participants. We then compared the number of participants who chose the best option as determined individually for each participant using a chi-square test. The proportion of participants in the feeling-focus condition that chose their best option $(M=66.7 \%$, $S E=11.4)$ was significantly higher than in the detail-focus condition $(M=22.2 \%, S E=10.1), \chi^{2}(1, N=36)=7.20, p<.01$. Thus, this finding replicates the finding from Experiment 1 that a focus on feelings versus details yields higher objective choice quality.

Finally, in order to examine whether the number of attributes recalled for each option differed between the conditions, an independent samples $t$ test was conducted on the percentage of recalled attributes. Participants in the feeling-focus condition did not recall more attributes $(M=20.4 \%, S E=2.1)$ than those in the detailfocus condition $(M=18.7 \%, S E=2.2), t(38)=.58, p>.5$. The differential focus of the two conditions, thus, did not influence the number of attributes that were later remembered.

In sum, Experiment 2 replicated the finding from Experiment 1 of higher objective decision quality for complex decisions in the feeling-focus versus detail-focus condition. More importantly, though, this study extended our investigation to include subjective decision quality, which was also significantly higher when participants focused on their feelings versus the details. Taken together, these results indicate that there are multiple benefits of focusing on feelings versus details during encoding for complex decisions. Importantly, these benefits are not due to deeper processing as reflected in differential memory encoding; participants recalled the same percentage of attributes irrespective of condition.

In contrast to the aim of this project examining attentional focus during encoding into working memory, as mentioned above, the work of Dijksterhuis and colleagues focuses on conscious versus unconscious processes that are at work after information acquisition. Thus, in order to further extend these findings with consideration of how different forms of conscious encoding interact with subsequent conscious versus unconscious thought, in Experiment 3 we expanded our procedures to include conditions that provided an opportunity for either subsequent conscious or unconscious thought.

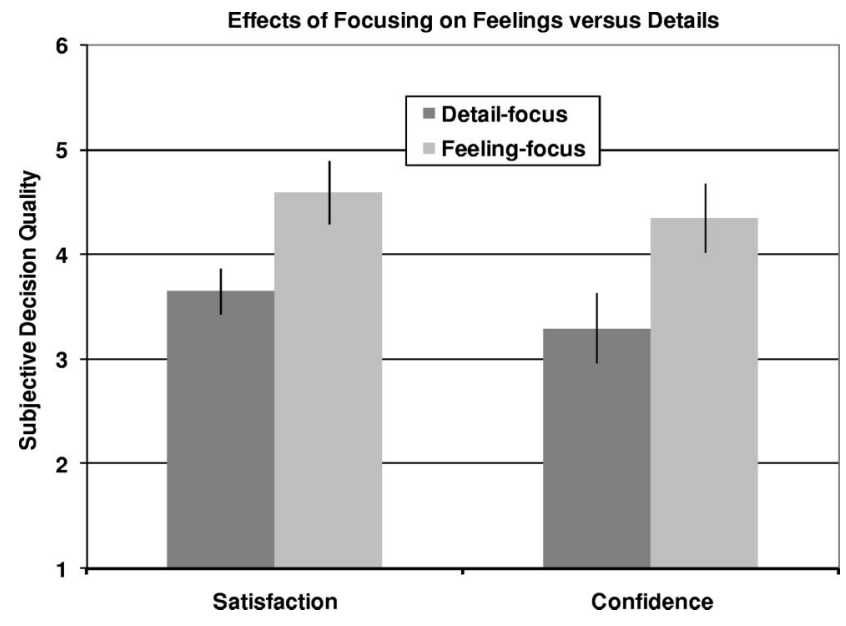

Figure 2. Mean satisfaction and confidence ratings in each condition. Error bars represent the standard error. 


\section{Experiment 3}

Although Experiments 1 and 2 provide evidence that focusing on feelings versus details can result in higher objective and subjective choice quality for complex decisions, the decisions were made immediately after information acquisition. Would the efficacy of affective versus deliberative encoding for complex decisions be diminished by subsequent conscious deliberation? Although UTT does not necessarily offer predictions beyond the subsequent benefits of unconscious thought for complex decisions, the affect heuristic offers the prediction that affective "tags" benefit time-limited decisions that may be made adequately with deliberative processes with additional time. Indirectly supporting this possibility, Wilson and Schooler (1991) demonstrated that introspection about affective appraisals of options can reduce choice quality. Moreover, McMackin and Slovic (2000) found that introspection about reasons impaired the quality of "intuitive" judgments but enhanced "analytic" judgments. Based on these findings, we expected that subsequent conscious deliberation would impair choice quality for feeling-focused but not detailfocused decisions. To test this hypothesis in Experiment 3, we manipulated participants' opportunity for subsequent conscious deliberation after either focusing on feelings or details during information acquisition.

\section{Method}

\section{Participants}

Seventy-six undergraduates $(M$ age $=20.07$ years; 56 females, 20 males) were randomly assigned to conditions in a 2 (feeling vs. detail focus) $\times 2$ (deliberation vs. distraction) betweenparticipants design. They received course credit for participation.

\section{Apparatus}

Same as Experiment 1.

\section{Design and Procedure}

The procedure was very similar to Experiment 1. Again participants were assigned to either a feeling-focus or detail-focus condition, but here only made complex decisions (four hypothetical cars, each defined by 12 attributes). In addition to experimentally manipulating attentional focus during encoding, we also included a subsequent deliberation manipulation. Modeled after the procedure of Djiksterhuis et al. (2006), participants were randomly assigned to conscious deliberation or distraction conditions. In the deliberation condition, participants were instructed to think about the decision for three minutes after information acquisition and prior to choosing a car. In the distraction condition, prior to making their choice participants were distracted for three minutes by completing a 2-back working memory task (Jonides et al., 1997). In the 2-back task, random digits (1-9) were presented serially for 2 seconds each. Participants were instructed to make a response if the current digit was the same digit that appeared two digits previously. After 3 minutes of conscious deliberation or distraction, participants chose one of the four cars.

\section{Results and Discussion}

We examined decision quality in the same manner as in Experiment 1; we compared the number of participants who chose the best option (the car with $75 \%$ positive attributes) using chi-square tests. As depicted in Figure 3, participants in the detail-focus condition preformed equivalently when given 3 minutes to deliberate over their decision (57.9\%) as when distracted for 3 minutes $(47.4 \%), \chi^{2}(1, N=38)=0.42, n s$. In contrast, in the feeling-focus condition, those participants who deliberated over the decision for 3 minutes chose an inferior option $(26.3 \%)$ more often that those who were distracted for 3 minutes $(57.9 \%), \chi^{2}(1, N=38)=3.89$, $p<.05$.

To verify that these effects could not be attributed to n-back performance as a potential confound, we examined accuracy on the n-back distractor task between the feeling-focus versus detailfocus groups. The groups performed the n-back with equivalent accuracy, $t(74)=1.15, n s$.

In this third study, we sought to examine how attentional focus during information acquisition interacted with subsequent deliberation. In Experiments 1 and 2, we found that focusing on feelings relative to the details resulted in higher choice quality for complex decisions when the choice was made immediately after all information was presented. In Experiment 3, we discovered that subsequent conscious deliberation relative to distraction led to lower decision quality in the feeling-focus conditions. In contrast, subsequent conscious deliberation did not influence quality in the detail-focus condition. Although Experiments 1 and 3 were separate investigations, examination of the patterns of performance across the two studies for the complex decisions suggests potential differences for delayed versus immediate decisions. It appears that choice performance in the detail-focus condition may benefit from the inclusion of a delay. These patterns suggest that a conscious detail-focused encoding strategy may benefit from additional processing time. However, it is clear in Experiment 3 that subsequent

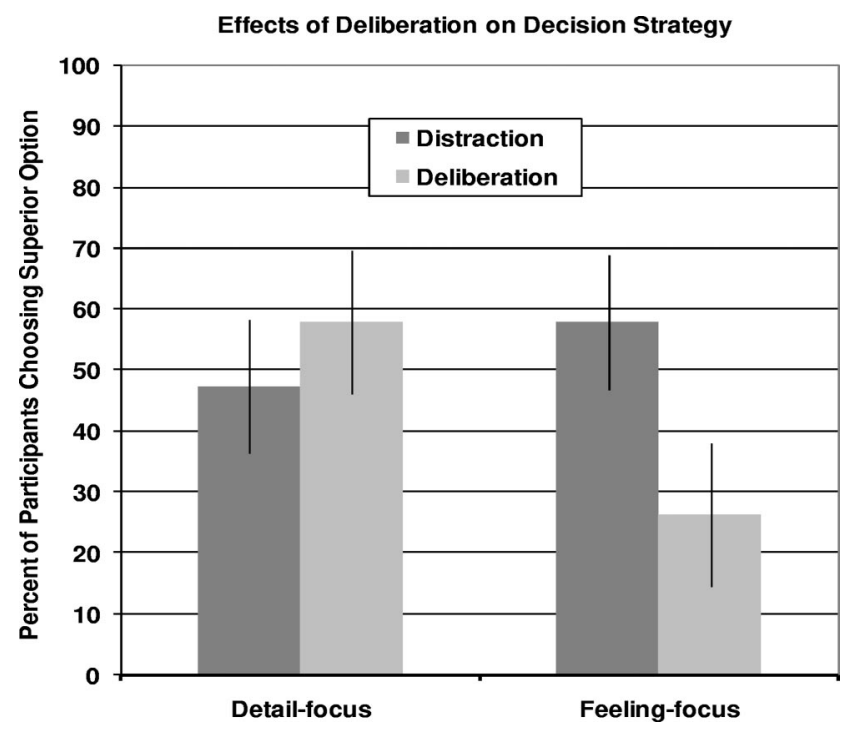

Figure 3. Percentage of participants in Experiment 3 who chose the best option (the car with $75 \%$ positive attributes) in each condition. Error bars represent the standard error. 
deliberation after conscious feeling-focused encoding impairs performance, whereas subsequent distraction does not. These patterns could then lead to the speculation that affective processes are perhaps somewhat more congruent with unconscious thought relative to deliberative processes.

Although these first three studies provide data demonstrating that feeling focused encoding is effective for complex decisions, there are a number of caveats that should be mentioned. First, the serial presentation of attributes is somewhat unnatural, as decisions are typically made while viewing and considering multiple attributes at a time. Second, our strategy reinforcement procedure (i.e., the intervening queries after each piece of information) was also somewhat unnatural. Finally, participants made only one decision in one domain (i.e., cars). In Experiment 4, we sought to address these methodological concerns while also extending the procedures to different domains.

\section{Experiment 4}

Given the aforementioned caveats to the above experiments, in Experiment 4, we again manipulated whether individuals were instructed to use an affective versus deliberative strategy, but with a less imposing manipulation and across four different domains (apartments, vacations, physicians, and medical treatments). In an attempt to provide greater generalizability for the previous results, we chose a broader and less imposing manipulation grounded in previous theory and research on intuitive/feeling-focused versus deliberative/reason-focused approaches to judgments (see, e.g., Epstein, 1994; Epstein, Donovan, \& Denes-Raj, 1999; Epstein et al., 1996). So as to encourage a more naturalistic strategy use, participants were instructed to rely on their gut feelings to guide their decisions versus using their considered, rational analysis to guide their decisions. These manipulations are broader than the feeling versus detailed focus manipulations of the previous studies and offer a less constrained and more robust interpretation and test of the affect heuristic; that is, the previous manipulations could be considered a specific operationally constrained approach for each of these general strategies as derived from theorizing on the affect heuristic. Also in this study, information was presented in a less resource-demanding grid format, and participants made multiple decisions. Finally, we included a control condition, in which participants were not given any particular strategy, in order to provide a basis of comparison for our strategy manipulations. Thus, the design of Experiment 4 represents an attempt to extend the previous findings, while also addressing various methodological constraints pertaining to the first three studies.

\section{Method}

\section{Participants}

Forty-six undergraduate students (28 female, 18 male) aged $18-21(M=19.76, S D=1.00)$ participated in exchange for course extra credit. The present study incorporated a 3 (strategy: feeling-focus vs. reason-focus vs. control) $\times 4$ (domain: apartments vs. vacations vs. physicians vs. treatments) withinparticipant factorial design.

\section{Apparatus}

Same as Experiment 1.

\section{Decision Grids}

For each decision, participants read information in a grid listing four options (e.g., Physician A, Physician B, Physician C, Physician D) in columns and 12 attributes in rows. Choice attributes were derived from pilot studies in which participants rated a variety of attributes, from which we selected the 12 attributes rated as most important. The options were constructed in a similar manner to Experiments 1 and 3 such that one option had 75\% positive attributes (the "best" option), two options had 50\% positive attributes, and the final option had $25 \%$ positive attributes.

In order to increase the difficulty of the decision task, values for the choice attributes were initially concealed from participants and were displayed one option (that is, one column with 12 pieces of information) at a time, in random order, for 5 seconds per option. After the last column was presented, participants indicated their choice.

\section{Procedure}

Participants completed a series of decisions using three strategies (feeling-focus, reason-focus, and no-strategy/control) in four domains (apartments, vacations, physicians, and treatments). All participants made four decisions (one for each of the four domains) using each of the three strategies, for a total of 12 decisions. As described above, strategy manipulations were adapted from previous research on intuitive/feeling-focused versus deliberative/ reason-focused approaches to judgments (see, e.g., Epstein, 1994; Epstein et al., 1999; Epstein et al., 1996). Specifically, participants were instructed to "rely on your gut feelings to guide your decisions" (feeling-focus), "use your considered, rational analysis to guide your decisions" (reason-focus), or were given no imposed strategy (control) prior to examining the decision grids. After reading information about the options for each decision, participants selected their preferred option and reported the extent to which they relied on their feelings or the details about the options to guide their decisions using a 7-point Likert-type scale (1- not at all to 7-extremely). Decisions were blocked by strategy such that participants made four decisions using a given strategy before making decisions with another strategy. To control for possible ordering effects, we randomized the order of decision blocks, as well as the order of decision domains within each block. Additionally, decision strategy blocks were separated by unrelated filler questionnaires so as to facilitate shifting strategies.

\section{Results and Discussion}

\section{Manipulation Check}

To verify that participants followed the strategy instructions in making their decisions, repeated-measures analyses of variance (ANOVA) were performed on the self-report measures of the extent to which they relied on their feelings or the details. As expected, participants reported greater reliance on feelings when using the feeling-focus strategy $(M=4.41, S D=1.24)$ than either the reason-focus $(M=3.48, S D=1.43)$ or control strategies $(M=$ $3.79, S D=1.43), F(2,90)=14.40, p<.001$. Also as expected, participants relied more on details when using the reason-focus strategy $(M=4.98, S D=1.11)$ than the feeling-focus $(M=4.44$, 
$S D=1.11)$ or control strategies $(M=4.55, S D=1.32), F(2$, 90) $=5.48, p<.01$.

\section{Decision Quality}

Choice quality was determined as the percentage of decisions for which participants chose the best option (with $75 \%$ positive attributes). A repeated-measures analysis of variance (ANOVA) using the within-participant variables focus type (feeling-focus, reason-focus, control/no focus) and domain type (apartment, vacation, physician, treatment) revealed a significant main effect of focus type on decision quality, $F(2,43)=3.30, p<.05$. Pairwise comparisons indicated that feeling-focus decisions resulted in higher choice quality $(M=82.6)$ than either reason-focus $(M=$ $75.5)$ or control decisions $(M=70.7), t \mathrm{~s}(45)=2.11 \& 2.33, p \mathrm{~s}<$ .05 , respectively. The quality of reason-focus decisions did not differ from control decisions, $t(45)=1.16, p>.2$.

The main effect of decision strategy on choice quality was qualified by a strategy by domain interaction, $F(6,39)=3.11, p<$ .05 (see Table 2). For decisions involving vacations, feeling-focus decisions were of higher quality than either reason-focus or control decisions, $t \mathrm{~s}(45)>3.0, p \mathrm{~s}<.005$. For decisions involving apartments, feeling-focus decisions were of higher quality than control decisions, $t(45)=3.08, p<.005$, but not reason-focus decisions, $t(45)=1.27, n$ s. No differences emerged in choice quality between decision strategies for physicians and treatments. Given that strategy was varied within participants in this study, analyses including strategy order as a factor resulted in no significant effects for order nor influenced the other results reported above.

Experiment 4 provides a more nuanced indication as to when feeling-focus strategies may be particularly beneficial. Specifically, an affective strategy was superior to a reason-based strategy for vacation but not the other domains. For apartments, an affective approach was superior to the control condition but was not significantly beneficial relative to the reason-focus condition, providing qualified support. Importantly, though, the procedures in Experiment 4 were significantly different from the previous experiments, which may have rendered the decisions in Experiment 4 less complex and difficult. For instance, for the "complex" decisions in this experiment, choice quality was overall at approximately $76 \%$ in contrast to choice quality in the prior three experiments, in which choice quality was overall at approximately $46 \%$. As such, the results of Experiment 4 may be less effective than the previous three studies in differentiating the benefits of affectiveversus reason-based strategies.

Table 2

Decision Quality by Strategy and Domain for Experiment 4

\begin{tabular}{lccc}
\hline \multicolumn{3}{c}{ Strategy } \\
\cline { 2 - 3 } \multicolumn{1}{c}{ Domain } & Feeling-focus & Reason-focus & Control \\
\hline Apartments & $91.3(4.2)$ & $82.6(5.7)$ & $65.2(7.1)$ \\
Vacations & $89.1(4.6)$ & $60.9(7.3)$ & $60.9(7.3)$ \\
Physicians & $78.3(6.1)$ & $82.6(5.7)$ & $78.3(6.1)$ \\
Treatments & $71.7(6.7)$ & $76.1(6.4)$ & $78.3(6.1)$ \\
Average & $82.6(2.6)$ & $75.5(2.7)$ & $70.7(4.1)$ \\
\hline
\end{tabular}

Note. Standard errors are in parentheses.

\section{General Discussion}

In the present investigation, we sought to examine the benefits of affect-focused decision making. Experiments 1 and 2 documented that focusing on feelings versus details during encoding resulted in higher objective and subjective decision quality for complex decisions.

In Experiment 3, focusing on feelings again resulted in superior choice quality, but only when subsequent conscious deliberation was precluded. Finally, the fourth experiment, which employed a significantly different methodology, provided qualified support for the relative benefits of using an affective strategy. Thus, these studies document that strategies drawing on affect are indeed beneficial, but with important constraints.

These findings extend the existing literature on intuitive decision making in a number of ways. First and foremost, these data provide a direct test of the efficacy of the affect heuristic across different decisions. Consistent with the theorizing of Slovic et al. (2002), basing decisions on affective impressions can be superior for certain complex decisions, contrary to traditional thinking that emotions interfere with decision making (for a review see Vohs, Baumeister, \& Loewenstein, 2007). Second, although previous studies have examined the benefits of subsequent unconscious processes for complex decisions (see, e.g., Dijksterhuis \& Nordgren, 2006), we know of no studies that have directly examined feeling-focus approaches to complex decision making. Theorizing on the affect heuristic has articulated the potential benefits of reliance on emotion, but few studies have directly tested this notion (e.g., Finucane et al., 2000). As such, the current report adds direct support for the benefits of feeling-focus decision strategies for complex decisions.

Both affective and deliberative processes play an important role in decision making, but the interaction of the two has been understudied. Although the work by Damasio (1994) underscores the importance of emotion to decision making and has shown that affective and deliberative processes are dissociable (Bechara, Damasio, Tranel, \& Anderson, 1998), little work has examined how the two modes of processing interact. Here we report data suggesting that deliberative processes may interfere with affective processes. Specifically, in Experiment 3, we found that participants who engaged in subsequent deliberation regarding the decision made lower quality choices relative to those who were distracted from subsequent conscious deliberation. This finding suggests that when using feeling-based encoding, it is best not to overthink the decision, consistent with previous work (McMackin \& Slovic, 2000; Wilson \& Schooler, 1991). While our findings complement those of Dijksterhuis and colleagues, they also suggest that affective encoding is superior to deliberative encoding for complex decisions-in a similar manner but distinct fromsubsequent unconscious versus conscious thought.

The distinction between encoding and later deliberation deserves further consideration. Constrained versus unconstrained encoding is one critical distinction between the current set of studies and previous work on unconscious thought. Dijksterhuis and Nordgren (2006) acknowledge the importance of conscious encoding as critical to the upstream effects of subsequent conscious or unconscious deliberation, but they do not differentiate the affective versus deliberative nature of this encoding. Under conditions in which encoding into working memory is not constrained 
(as in their research paradigms), it is very likely that information is encoded by both affective and cognitive processes. Thus, the work of Dijksterhuis and colleagues addresses "intuition" from the perspective of subsequent processing and whether this processing is conscious or unconscious. However, examining the way in which attentional focus on emotion versus details influences the weight of affective tags represents an exciting direction for future studies.

Similarly, Lassiter et al. (2009) draw the distinction between online and memory-based processing without consideration of affect. Importantly though, the current study focuses on cognitive versus affective encoding into working memory in an online manner, whereas Lassiter et al. focused on online encoding into working memory versus encoding into long-term memory. Interestingly, in Experiment 3, we found that 3 minutes of deliberation after feeling-based encoding impaired decision-quality, while 3 minutes of deliberation were beneficial for detail-focused encoding. This line of reasoning suggests that the online condition in the work of Lassiter et al. (2009) might involve affective encoding, but such speculation requires future research. Here we report data related to specific conscious encoding strategies. Thus, while our findings cannot be directly compared to those of Dijksterhuis and Lassiter, they are complementary in suggesting that affective processes may be at work in the "gut feeling" considered a cornerstone for intuition. Future studies will be necessary, however, to precisely delineate the intersection of affective and unconscious processes.

Given recent attention to ecological rationality (e.g., Gigerenzer, 2008; Todd \& Gigerenzer, 2007), we examined different domains, and found that affective strategies may prevail only for certain domains. Importantly, these considerations are qualified by significant methodological changes between the experiments. Despite the differences in procedure, though, the benefits of an affective strategy emerged for cars and vacations, partially for apartments, but not for physicians and treatments. For college students, these former domains may be more familiar or more important and salient. Coupled with the aforementioned research showing that experts rely to a greater extent on intuitive processes when making decisions (Reyna, 2004; Reyna \& Lloyd, 2006), Dijksterhuis, Bos, van der Leij, \& van Baaren (in press) recently demonstrated that experts make better predictions after a period of unconscious versus conscious thought. These lines of research suggest that familiarity and/or greater expertise may explain the current domain effect in that decisions about cars, vacations, and apartments are more familiar to college students than decisions about physicians and treatments. However, it is also possible that the observed domain differences were due to these domains being more "affective" versus "rational" by nature (Inbar, Cone, \& Gilovich, 2010). That is, it remains equally plausible that the former domains are more affectively driven; these domains maybe intrinsically more amenable to affective versus deliberative strategies. Finally, as described above, the methodological differences in Experiment 4 may have influenced the results. Disentangling these different possibilities represents an important area for future investigation.

While the current studies report on the benefits of emotionbased strategies, it remains possible that the detail-focus condition resulted in inferior choice quality due to the slow and serial nature of such processes, or due to distracting attention away from feelings. This distinction regarding the detail-focus condition repre- sents another direction for future research. Additionally, as described above, there are certainly instances when emotion may lead to flawed or biased decisions (Gilovich et al., 2002), which importantly can be corrected with deliberation (see, e.g., Small, Loewenstein, \& Slovic, 2007). For instance, there appears to be an affective component that leads to the framing effect (Cheung \& Mikels, in press), in which the presentation of alternatives as gains or losses for equivalent expected outcomes results in biased asymmetric decisions (i.e., risk aversion for gains and risk seeking for losses). Thus, given the dearth of such investigations, future work will be needed to precisely delineate when emotion benefits versus harms decision making.

In sum, the results of the current investigation suggest that affective strategies are indeed an effective means to making good decisions, thus contributing to findings on the benefits of affect in decision making. Moreover, the results are prescriptive with respect to when and how such strategies should be used. While many open questions remain, the current results support the notion that when the going gets tough, go with your gut-but with the qualification that one should not overthink their decision.

\section{References}

Acker, F. (2008). New findings on unconscious versus conscious thought in decision making: Additional empirical data and meta-analysis. Judgment and Decision Making, 3, 292-303.

Alhakami, A. S., \& Slovic, P. (1994). A psychological study of the inverse relationship between perceived risk and perceived benefit. Risk Analysis, 14, 1085-1096.

Bechara, A., Damasio, H., Tranel, D., \& Anderson, S. W. (1998). Dissociation of working memory from decision making within the human prefrontal cortex. Journal of Neuroscience, 18, 428-437.

Breckler, S. J. (1984). Empirical validation of affect, behavior, and cognition as distinct components of attitude. Journal of Personality and Social Psychology, 47, 1191-1205.

Breckler, S. J., \& Wiggins, E. C. (1989). Affect versus evaluation in the structure of attitudes. Journal of Experimental Social Psychology, 25, 253-271.

Brownstein, A. L. (2003). Biased predecision processing. Psychological Bulletin, 129, 545-568.

Cheung, E., \& Mikels, J. A. (in press). I'm feeling lucky: The relationship between affect and risk-seeking in the framing effect. Emotion.

Crites, S. L., Fabrigar, L. R., \& Petty, R. E. (1994). Measuring the affective and cognitive properties of attitudes: Conceptual and methodological issues. Personality and Social Psychology Bulletin, 20, 619-634.

Damasio, A. R. (1994). Descartes' error: Emotion, reason, and the human brain. New York: Avon Books.

Denes-Raj, V., \& Epstein, S. (1994). Conflict between intuitive and rational processing: When people behave against their better judgment. Journal of Personality and Social Psychology, 66, 819-829.

Dijksterhuis, A. (2004). Think different: The merits of unconscious thought in preference development and decision making. Journal of Personality and Social Psychology, 87, 586-598.

Dijksterhuis, A., Bos, M. W., Nordgren, L. F., \& van Baaren, R. B. (2006). On making the right choice: The deliberation-without-attention effect. Science, 311, 1005-1007.

Dijksterhuis, A., \& Nordgren, L. F. (2006). A theory of unconscious thought. Perspectives on Psychological Science, 1, 95-109.

Dijksterhuis A., Bos M. W., van der Leij, A., \& van Baaren, R. B. (in press). Predicting soccer matches after unconscious and conscious thought as a function of expertise. Psychological Science.

Epstein, S. (1994). Integration of the cognitive and the psychodynamic unconscious. American Psychologist, 49, 709-724. 
Epstein, S., Donovan, S., \& Denes-Raj, V. (1999). The missing link in the paradox of the Linda conjunction problem: Beyond knowing and thinking of the conjunction rule, the intrinsic appeal of heuristic processing. Personality and Social Psychology Bulletin, 25, 204-214.

Epstein, S., Pacini, R., Denes-Raj, V., \& Heier, H. (1996). Individual differences in intuitive-experiential and analytical-rational thinking styles. Journal of Personality and Social Psychology, 71, 390-405.

Evans, J. S. B. T. (2008). Dual-processing accounts of reasoning, judgment, and social cognition. Annual Review of Psychology, 59, 255-278.

Finucane, M. L., Alhakami, A., Slovic, P., \& Johnson, S. M. (2000). The affect heuristic in judgments of risks and benefits. Journal of Behavioral Decision Making, 13, 1-17.

Gigerenzer, G. (2008). Why heuristics work. Perspectives on Psychological Science, 3, 20-29.

Gilovich, T., Griffin, D., \& Kahneman, D. (Eds.). (2002). Heuristics and biases: The psychology of intuitive judgment. New York: Cambridge University Press.

González-Vallejo, C., Lassiter, G. D., Bellezza, F. S., \& Lindberg, M. J. (2008). "Save angels perhaps": A critical examination of unconscious thought theory and the deliberation-without-attention effect. Review of General Psychology, 12, 282-296.

Hsee, C. K. (1996). The evaluability hypothesis: An explanation for preference reversals between joint and separate evaluations of alternatives. Organizational Behavior and Human Decision Processes, 67, 247-257.

Hsee, C. K. (1998). Less is better: When low-value options are valued more highly than high-value options. Journal of Behavioral Decision Making, 11, 107-121.

Hsee, C. K., \& Rottenstreich, Y. (2004). Music, pandas, and muggers: On the affective psychology of value. Journal of Experimental Psychology: General, 133, 23-30.

Inbar, Y., Cone, J., \& Gilovich, T. (2010). People's intuitions about intuitive insight and intuitive choice. Journal of Personality and Social Psychology, 99, 232-247.

Janis, I. L., \& Mann, L. (1977). Decision making: A psychological analysis of conflict, choice, and commitment. New York: The Free Press.

Jonides, J., Schumacher, E. H., Smith, E. E., Lauber, E. J., Awh, E., Minoshima, S., \& Koeppe, R. A. (1997). Verbal working memory load affects regional brain activation as measured by PET. Journal of Cognitive Neuroscience, 9, 462-475.

Kahneman, D. (2003). A perspective on judgment and choice: Mapping bounded rationality. American Psychologist, 58, 697-720.

Lassiter, G. D., Lindberg, M. J., Gonzalez-Vallejo, C., Bellezza, F. S., \& Phillips, N. D. (2009). The deliberation-without-attention effect: Evidence for an artifactual interpretation. Psychological Science, 20, 671675

Lerouge, D. (2009). Evaluating the benefits of distraction on product evaluations: The mind-set effect. Journal of Consumer Research, 36, 367-379

Loewenstein, G. F., Weber, E. U., Hsee, C. K., \& Welch, N. (2001). Risk as feelings. Psychological Bulletin, 127, 267-286.

McMackin, J., \& Slovic, P. (2000). When does explicit justification impair decision making? Applied Cognitive Psychology, 14, 527-541.

Mikels, J. A., Loeckenhoff, C. E., Maglio, S. J., Goldstein, M. K., Garber, A., \& Carstensen, L. L. (2010). Following your heart or your head: Focusing on emotions versus information differentially influences the decisions of younger and older adults. Journal of Experimental Psychology: Applied, 16, 87-95.
Mikels, J. A., Reuter-Lorenz, P. A., Beyer, J. A., \& Fredrickson, B. L. (2008). Emotion and working memory: Evidence for domain-specific processes for affective maintenance. Emotion, 8, 256-266.

Millar, M. G., \& Tesser, A. (1986). Effects of affective and cognitive focus on the attitude-behavior relation. Journal of Personality and Social Psychology, 51, 270-276.

Peters, E., Hess, T. M., Vastfjall, D., \& Auman, C. (2007). Adult age differences in dual information processes: Implications for the role of affective and deliberative processes in older adults' decision making. Perspectives on Psychological Science, 2, 1-23.

Reyna, V. F. (2004). How people make decisions that involve risk: A dual-processes approach. Current Directions in Psychological Science, $13,60-66$.

Reyna, V. F., \& Lloyd, F. J. (2006). Physician decision making and cardiac risk: Effects of knowledge, risk perception, risk tolerance, and fuzzy processing. Journal of Experimental Psychology: Applied, 12, 179-195.

Shiv, B., Loewenstein, G., Bechara, A., Damasio, H., \& Damasio, A. R. (2005). Investment behavior and the negative side of emotion. Psychological Science, 16, 435-439.

Slovic, P., Finucane, M., Peters, E., \& MacGregor, D. G. (2002). The affect heuristic. In T. Gilovich, D. Griffin, \& D. Kahneman (Eds.), Heuristics and biases: The psychology of intuitive judgment. (pp. 397-420). New York: Cambridge University Press.

Slovic, P., Finucane, M. L., Peters, E., \& MacGregor, D. G. (2004). Risk as analysis and risk as feelings: Some thoughts about affect, reason, risk, and rationality. Risk Analysis, 24, 311-322.

Slovic, P., Peters, E., Finucane, M. L., \& MacGregor, D. G. (2005). Affect, risk, and decision making. Health Psychology, 24, S35-S40.

Small, D. A., Loewenstein, G., \& Slovic, P. (2007). Sympathy and callousness: The impact of deliberative thought on donations to identifiable and statistical victims. Organizational Behavior and Human Decision Processes, 102, 143-153.

Stanovich, K. E., \& West, R. F. (2000). Individual differences in reasoning: Implications for the rationality debate? Behavioral and Brain Sciences, 23, 645-726.

Todd, P. M., \& Gigerenzer, G. (2007). Environments that make us smart: Ecological rationality. Current Directions in Psychological Science, 16 167-171.

Trafimow, D., \& Sheeran, P. (1998). Some tests of the distinction between cognitive and affective beliefs. Journal of Experimental Social Psychology, 34, 378-397.

van den Berg, H., Manstead, A. S. R., van der Pligt, J., \& Wigboldus, D. H. J. (2006). The impact of affective and cognitive focus on attitude formation. Journal of Experimental Social Psychology, 42, 373-379.

Vohs, K. D., Baumeister, R. F., \& Loewenstein, G. (Eds.). (2007). Do emotions help or hurt decision making? A Hedgefoxian perspective. New York: Russell Sage Foundation.

Wilson, T. D., Lisle, D. J., Schooler, J. W., Hodges, S. D., Klaaren, K. J., \& LaFleur, S. J. (1993). Introspecting about reasons can reduce postchoice satisfaction. Personality and Social Psychology Bulletin, 19, 331-339.

Wilson, T. D., \& Schooler, J. W. (1991). Thinking too much: Introspection can reduce the quality of preferences and decisions. Journal of Personality and Social Psychology, 60, 181-192.

Received November 12, 2009

Revision received March 31, 2011

Accepted April 7, 2011 